\title{
Finding a new target in an old display: Evidence for a memory recency effect in visual search
}

\author{
Christof KöRNER \\ University of Graz, Graz, Austria \\ AND \\ IAIN D. GILCHRIST \\ University of Bristol, Bristol, England
}

\begin{abstract}
Visual search often involves searching the same environment, consecutively, for a number of different targets. Here we investigate the extent to which search benefits from such previous exposure. In the experiment participants searched the same display consecutively for two different targets. Manual responses were faster in the second search than the first search regardless of whether a target was present or absent in the second search. Eye movement recordings demonstrated that the time necessary to find a target letter in the second search depended on when that letter was last fixated in the previous search. This fixation recency effect lasted for about four fixations. In addition, when a target was absent during the second search, participants were less likely to refixate a distractor if it had been recently fixated in the previous search and refixations tended to also occur later on in the search. These results provide evidence for a limited capacity short-term memory store in this kind of visual search.
\end{abstract}

The visual environment contains multiple objects most of which are irrelevant to the current behavioral goal. The task for any organism is to locate and identify the item that is relevant (the target) from among the other objects (the distractors). In a complex environment this task involves visual search, a topic that has attracted a great deal of research effort over the last 30 years. This research focuses on situations in which, for each search participants are presented with a whole new display. Of course, in real life visual behavior occurs in a world that is far more continuous. We search for a number of things, one after the other, within the same environment. Only recently has this kind of repeated search attracted some attention (Wolfe, Klempen, \& Dahlen, 2000; Wolfe, Oliva, Butcher, \& Arsenio, 2002). Wolfe and colleagues were interested in the longterm benefits of repeated exposure on search performance. Their results indicate that participants do not profit from prior search experience in the longer term. This, however, does not exclude the possibility of a short-lived influence of visual short-term memory on search behavior which we explore in the present article.

Repeated search is intrinsically interesting because it maps readily onto everyday behavior. However, it is also of interest because of the recent debate about the extent to which visual search is supported by memory (see Shore $\&$ Klein, 2000). Since the initial report by Horowitz and Wolfe (1998) that there is no memory involvement in vi- sual search, a number of groups have produced empirical evidence for memory mechanisms that prevent item revisits (e.g., Boot, McCarley, Kramer, \& Peterson, 2004; Kristjansson, 2000; Peterson, Kramer, Wang, Irwin, \& McCarley, 2001) while others have emphasized the limited capacity of these memory processes (Gilchrist \& Harvey, 2000; Körner \& Gilchrist, in press; McCarley, Wang, Kramer, Irwin, \& Peterson, 2003). However, what still remains unclear is the exact properties of any memory processes that may support visual search.

Within the classical memory literature, memory is divided into short-term and long-term (Atkinson \& Shiffrin, 1968). Short-term memory is said to have a limited capacity and to decay over time. The classical signature of short-term memory is the recency effect: Items that have been processed more recently have a higher probability of being recalled (e.g., McCrary \& Hunter, 1953). The recency effect reflects both the limited capacity and the rapid decay of short-term memory. If such memory processes do indeed support search then we would expect to observe these effects in situations in which search occurs repeatedly in the same display for different targets. In such repeated search, any memory resources available will confer a real advantage for subsequent searches.

With this motivation, in the present experiment we asked participants to carry out two consecutive searches on the same display and looked for evidence of short-term

C. Körner, christof.koerner@uni-graz.at 
memory for the display items. In this search paradigm participants received auditory information about which target letter to search for and made a present-absent manual response. When they had responded to the first target they receive a new, second, target letter to search for. Participants search the same unaltered display for both targets. Note that in this paradigm the letter that becomes a target was a distractor in the preceding search. If the time necessary to respond to the new target letter depends on when this letter was last processed before it became the target this will be evidence of a classical memory recency effect.

\section{METHOD}

\section{Design}

Participants searched the same 10-letter display twice for different target letters. In both searches the target was present on half the trials and absent on the remainder resulting in four conditions: absent-absent (AA), absent-present (AP), present-absent (PA), and present-present (PP). We recorded participants' manual response times (RTs) and their eye movements.

\section{Participants}

Eight students ( 7 female) from the Department of Psychology, University of Graz, participated for course credit. The mean age was 22.0 years (range 18 to 27). All participants had normal or corrected-to-normal vision.

\section{Stimuli and Procedure}

Stimuli were presented in white on a black background. In each trial, we displayed ten uppercase letters in Arial font which subtended $0.32^{\circ}$ at the viewing distance of $63 \mathrm{~cm}$. A white circle surrounded each letter. The outer diameter of the circle was $0.9^{\circ}$ and the circle was $0.18^{\circ}$ thick (see Figure 1). The letters were sampled ran- domly from the list of the following 12 letters: A, E, F, G, H, K, M, $\mathrm{O}, \mathrm{R}, \mathrm{S}, \mathrm{W}$, and $\mathrm{X}$. The circles around the letter served two purposes: (1) They acted to reduce the ability to identify the letter without fixation (cf. Bouma, 1970), and (2) they provided a clear target for the saccadic orienting. In a pilot experiment, we demonstrated that letter identification did not differ reliably from chance when fixation was more than $3^{\circ}$ away from these stimuli.

The 10 letters were positioned at the intersections of an imaginary $6 \times 6$ grid. The size of a grid cell was $3.6^{\circ}$. The letter position deviated randomly from the intersection $\pm 0.23^{\circ}$ both in horizontal and vertical direction. The whole viewing area subtended $21.6^{\circ} \times 21.6^{\circ}$ (see Figure 1).

Each observer participated in eight blocks of 64 trials (spread over two days) resulting in a total of 128 trials per condition per participant. At the beginning of each trial a fixation disc was presented and remained present until fixation was registered at that location. The disc was presented at a position chosen randomly from the locations where a letter would appear in the search display. When fixation was registered a placeholder display was presented. It was identical to the search display except that each letter was replaced by the hash symbol (\#). After $500 \mathrm{msec}$ the placeholder display was replaced by the search display (search display onset). Simultaneously to search display onset, the first target letter was announced through loudspeakers placed to the left and the right of the display monitor. For target present searches, the letter with the smallest deviation from the distance of $10.8^{\circ}$ from the fixation disc in the display was selected as the target. This distance corresponded to three times the size of a cell of the underlying $6 \times 6$ grid. As soon as the participant pressed a button in response to the first target the name of the second target letter was announced. For target present searches the letter with the smallest deviation from the distance of $10.8^{\circ}$ from the last fixation in Search 1 was selected as the target. If, in the PP condition, the determined target for Search 2 was identical to the Search 1 target, a different target letter was selected at random. When the participant responded to the second target the display was cleared. From search display onset until a participant's response to the second target the display remained unchanged. Participants were instructed to press

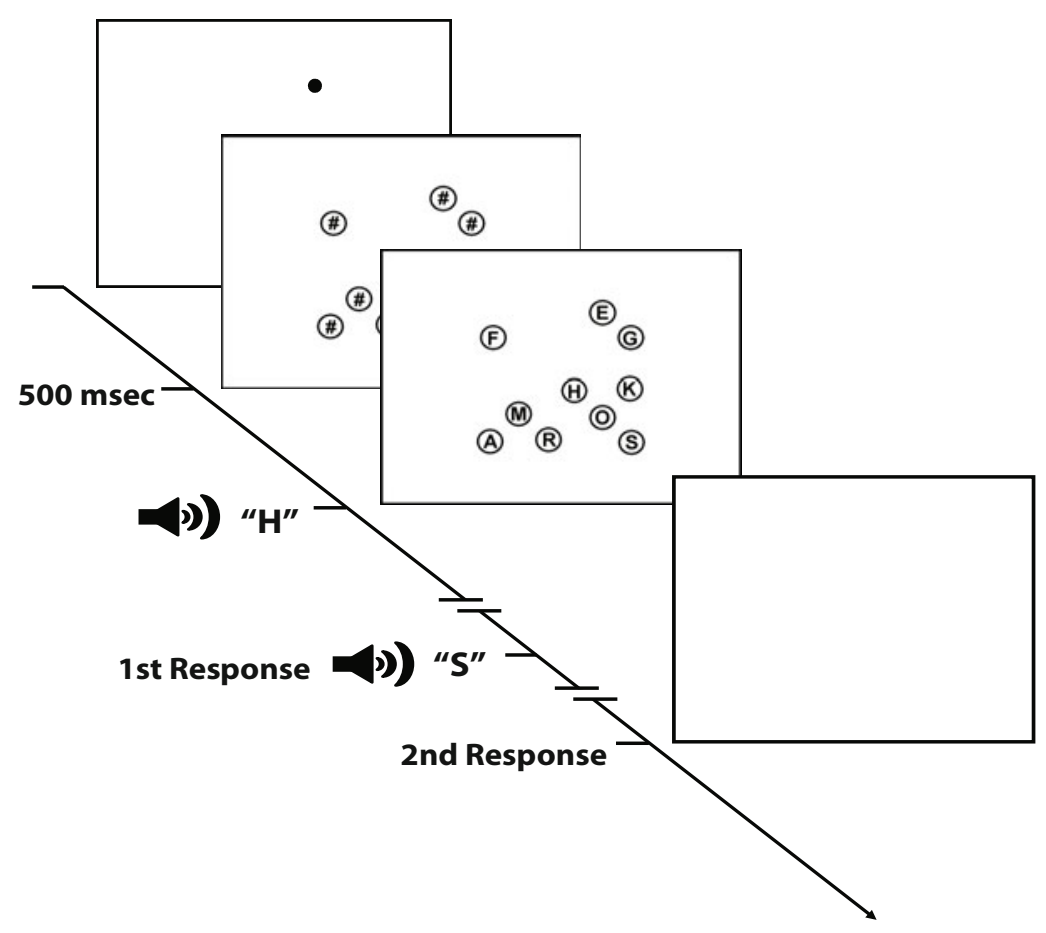

Figure 1. Sequence of events in a trial. 
the right button on a response box for a target-present response and the left button for an absent response; they were told to respond as quickly and as accurately as possible.

The sequence of trials within a block was chosen randomly for each participant. Participants were given a break of approximately 5 min between blocks.

\section{Apparatus}

We recorded two-dimensional eye movements using an EyeLink II eyetracker (SR Research, Canada). We recorded from both eyes at a sampling rate of $250 \mathrm{~Hz}$ and analyzed the data from the eye that produced the best spatial resolution, which was typically better than $0.35^{\circ}$. Displays were presented on a 21 -in. monitor with a resolution of $1,152 \times 864$ pixels. A chinrest was used to minimize head movement.

\section{RESULTS}

We obtained data from 4,096 trials ( 8 participants $\times$ 512 trials). Due to computer error, there were 186 trials $(4.4 \%)$ that could not be included in the analysis. A further $34(0.9 \%)$ were excluded for the PP condition when the second target was selected at random instead of according to distance (see Procedure). The remaining 3,876 trials were analyzed.

\section{Manual Responses}

Incorrect trials on either one of the searches were excluded from further analyses. On average $3.2 \%$ errors occurred with a range across participants of $0.8 \%$ to $5.9 \%$. The maximum error rate across conditions was $5.8 \%$ (condition PP).

Figure 2 shows the mean correct RT for each condition (averaged across individual means). Averaged across search target-present search was reliably faster than targetabsent search $(2,070 \mathrm{msec}$ and 3,608 msec, respectively) $[F(1,7)=135.3, p<.01]$, and, averaged across target presence, Search 2 was faster than Search $1(2,754 \mathrm{msec}$ and $2,924 \mathrm{msec}$, respectively) $[F(1,7)=10.2, p<.05]$. There was no interaction. Besides a typical effect of target presence, Search 2 performance was faster than Search 1 performance.

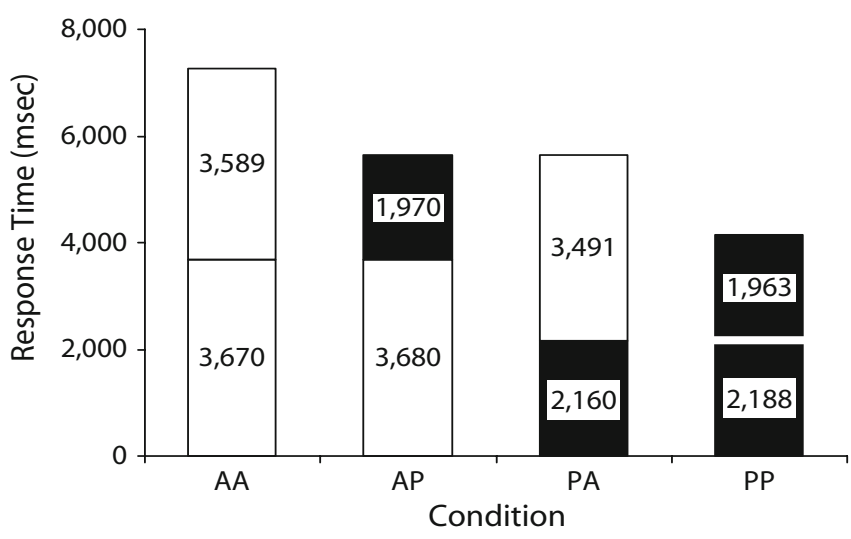

Figure 2. Mean response times (RTs) for each condition. Bottom bars denote Search 1 RTs. Top bars denote Search 2 RTs. White bars denote target-absent search trials, and black bars denote target-present search trials.

\section{Eye Movements}

To examine the origin of the search benefit found in Search 2, we analyzed conditions for which a target was present during Search 2 (i.e., conditions AP and PP). There were 881 correct trials for the AP condition and 868 correct trials for the PP condition. From these trials, we selected those for which the eyetracker had registered a fixation of the Search 2 target within the fixation sequence of Search 1 . This was the case for 721 AP trials and for 307 PP trials. For these trials, we determined the target recency position. This is the number of fixations in Search 1 between the last fixation in Search 1 and the fixation on the target in Search 2. For example, a recency of 1 means that a participant was fixating the Search 2 target just one fixation before he or she responded manually. Thus, for each trial, we obtained the recency of the Search 2 target within Search 1.

Figure 3 shows the time necessary to find the Search 2 target depending on its recency in Search 1, separately when a target was absent in Search 1 (condition AP, dotted line) and for a target being present in Search 1 (condition $\mathrm{PP}$, solid line). To investigate whether there is a reliable difference in Target 2 search time depending on target presence and target recency we conducted a $2 \times 9$ ANOVA using Search 1 target presence (absent vs. present) and recency (Positions 1-9) as factors and Search 2 RT as dependent measure. There was no effect of target presence $[F(1,7)<1]$; however, target recency had an effect on RT $[F(8,56)=3.43, p<.01]$. There was no interaction.

Given that target presence had no differential impact on Search 2 performance, for the subsequent analysis we pooled the data from conditions AP and PP. Figure 4 (circle plotting symbols) shows the relationship between search performance (manual RT) and target recency in the previous search for these pooled data. Search performance in Search 2 was faster when the target had been fixated more recently in Search 1.

To evaluate the recency benefit we compared the individual mean RT with the individual mean RT from trials in which a target was present in Search 1. This baseline is indicated by the dotted line in Figure 4. In a series of paired samples $t$ tests for each recency position, we found that performance for Recency Positions 1, 2, 3, and 5 was significantly better than baseline ( $p<.05$ in each case). The RTs for the other recency positions were not distinguishable from the baseline. This is a memory recency effect: Participants responded faster to a target when they had processed the item that became the target more recently.

The recency analysis presented here is subject to a number of potential problems. One major issue is that first searches of different lengths contribute differently to the different recency positions. For example, higher recency positions could occur only when participants searched for longer in Search 1, whereas low recency positions could occur on almost any trial. To control for this confound, we repeated this analysis with a subset of the data. We selected only trials with 10 or more fixations in Search 1 and recomputed the recency curve for Recency Positions 1 to 9; thus, all of the selected trials could contribute to any recency position. On average, data from 76.0 trials were 


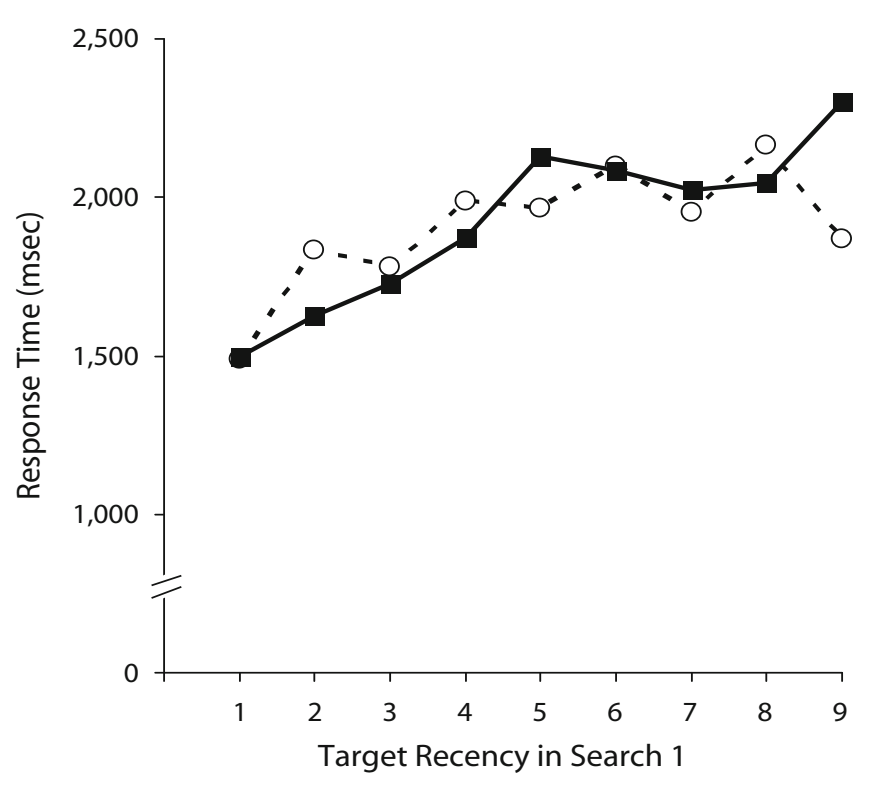

Figure 3. Mean response times for target-present trials in Search 2, separated for Search 1 absent (condition AP, dotted line) and Search 1 present (condition PP, solid line), depending on the recency of the target fixation in Search 1.

available for each recency position, and the number of trials available for any recency position did not deviate from this mean $[t(8)<1]$. For this subset of trials, the average number of fixations in Search 1 was 13.3 and did not differ across recency positions $[F(8,56)<1]$. Figure 4 plots the recency effect for this subset of data (square plotting symbols) and also for the whole data set (circle plotting symbols); the recency curves are almost identical. Again, for this subset of data, we found that performance for recency positions of $1,2,3$, and 5 was significantly better than baseline ( $p<.05$ in each case).

A straightforward explanation for the recency effect may be that participants subsubvocally rehearse the most recent items of Search 1. If this were the case, we would expect participants to respond straightaway if the newly announced target was in the rehearsed set, and we may not expect fixation of the target to occur. The probability of making a correct response on Search 2 without fixating the target should increase when the Search 2 target was fixated recently during Search 1 . However, we found that participants fixated the second target in $93.5 \%$ (range: $88.0 \%-97.6 \%$ ) of the correct trials. This rate mirrored an equally high percentage of $88.5 \%$ (range: $74.9 \%-95.0 \%$ ) of Search 1 target fixations. The small remainder of correct responses without target fixation left no room for variation depending on recency. If, on the other hand, the type of memory responsible for the recency effect is more visual rather than verbal, the time to fixate the target in Search 2 should vary as a function of recency, just as manual RT does (see Figure 4). When we computed the number of fixations until target fixation as a function of recency the resulting curve looked much like the ones depicted in Figure 4. There was a monotonic increase from 4.97 up to 6.96 fixations for the first four recency posi- tions before the number of fixations was indistinguishable from baseline (7.35 fixations before Search 1 target fixation). These analyses strongly suggest that verbal memory does not play a role here. However, it is not possible to rule this out completely and further empirical research, beyond the scope of the present report, would be needed to fully address this issue.

So far, we have focused on data from target present trials in Search 2. The recency effect suggests that participants are able to remember the identity and location of a recently fixated item such that they can find that item more quickly once it becomes a target. However, we also observed a benefit from Search 1 to Search 2 for target absent trials (see Figure 2). This benefit cannot be a result of returning to the new target. It seems more plausible that, if recently fixated items are retained, then this set may not need to be searched in order to produce an absent response. If that were the case, recently fixated items from Search 1 would be less likely to be (re)inspected during Search 2. To test this possibility, we analyzed data from 1,015 trials with targets absent in both searches (condition AA). For each participant and Recency Positions 1-5, we calculated the probability (relative frequency) of refixation of an item from Search 1 during Search 2.

Figure 5 (solid line) shows that this probability (averaged across participants) increased monotonically indicating that recently fixated items were often not reinspected before a participant responded. For all the items that were actually refixated, we computed the number of fixations before refixation in Search 2. If recent items are remem-

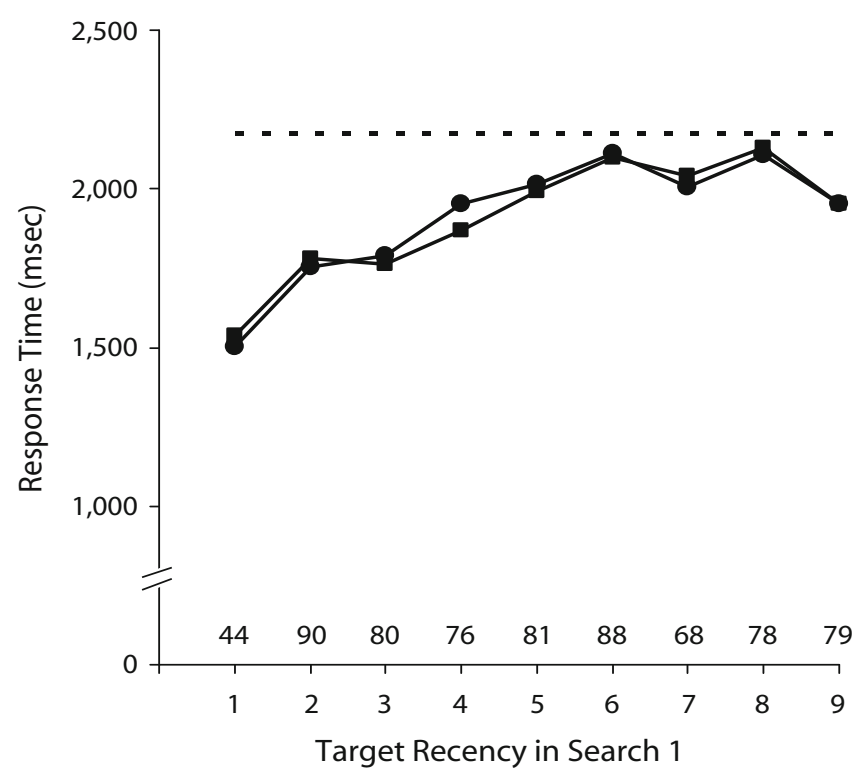

Figure 4. Mean response times (RTs) for target-present trials in Search 2 (pooled across conditions AP and PP), depending on the recency of the target fixation in Search 1 for the full data set (circle plotting symbols) and for data from a subset of trials with 10 or more fixations (square plotting symbols). The dotted line represents the baseline target-present $R T$ in Search 1 . The number of trials from the subset contributing to each recency position is indicated above the abscissa. 


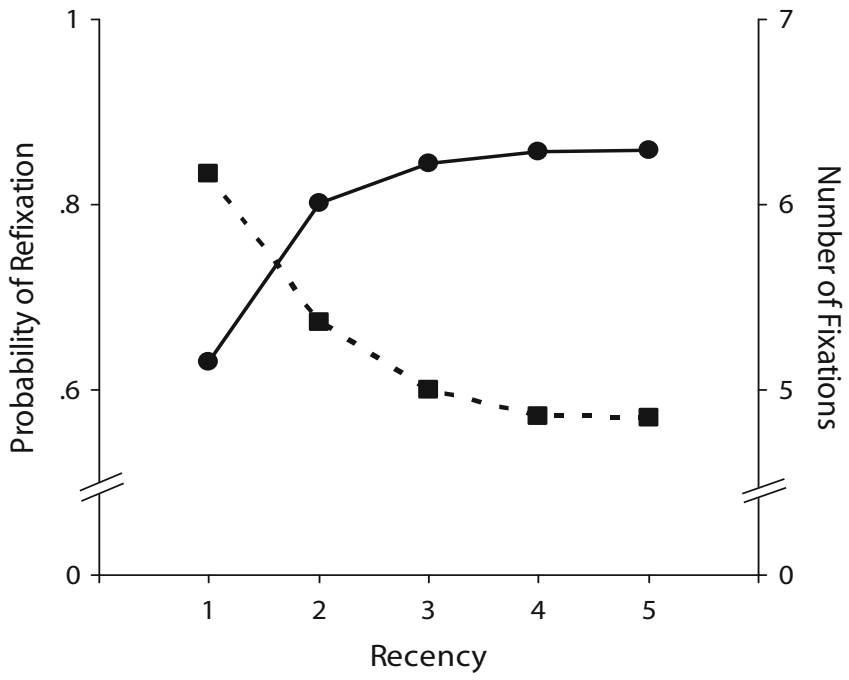

Figure 5. Probability of refixating an item during Search 2 (solid line) and number of fixations before refixation (dotted line), depending on that item's recency in Search 1 (condition AA).

bered but reinspected anyway, we would expect this to take longer before they are being refixated. Figure 5 (dotted line) shows that the average number of fixations before refixation decreases monotonically from about six to less than five fixations, indicating an inverse recency effect; recently inspected items are reprocessed later than are other items.

\section{DISCUSSION}

The results of this experiment provide a clear picture of some of the properties of the memory processes that support repeated visual search in a stable environment. The first search, and associated exposure to the display, improved performance in the second search.

For target present trials, the magnitude of this improvement depended on when the letter that is about to become the target in the next search was last fixated (i.e., its recency). The more recently an item was fixated in the preceding search the faster it could be found when it became the target. For less recently fixated items, beyond about five items, there was no measurable benefit; performance was indistinguishable from baseline. This pattern is surprisingly similar to the classic serial position recency function. A memory span of approximately four items is also consistent with recent estimates from research in visual search (McCarley et al., 2003) and in memory research (Cowan, 2001).

For target absent trials, the recently fixated items in the first search tend to be avoided in the subsequent search. When these items are fixated, this tends to occur later on in the second search presumably after the memory representation for them has decayed. Again, this pattern of data is consistent with a short-term memory system that has a limited capacity. This avoidance of recently visited locations is similar to the behavior that has previously been described as inhibition of return (Klein \& McInnes,
1999). However, in the present experiment, if the avoidance of previously visited locations were automatic we would expect this to lead to an elevated error rate in target present trials. The present data are more consistent with a single memory process leading to both the avoidance of recently fixated distractors and the rapid localization of recently fixated targets.

In our experiment, observers searched the same environment consecutively for different targets. This adapted search paradigm more closely reflects everyday search; we often search the same environment for different objects. This paradigm also allowed us to study what kind of memory processes support such search. Previous studies of more traditional single search have indicated that shortterm memory is involved in facilitating search (see Gilchrist \& Harvey, 2000; McCarley et al., 2003). However, in this kind of search, there is no functional advantage in remembering the identity of a distractor. Search could proceed efficiently on the basis of a memory representation that simply tags locations as visited without encoding the identity of the distractor at that location. Note, however, that such a tagging process would not results in the benefits reported in the present experiment. The benefits reported here would only occur if the identity of the distractor is encoded during search. In the current experiment we demonstrate such an advantage which suggests that, at least in these repeated search paradigms, the identity of the distractor is encoded.

Whereas many researchers agree that there is a role for memory in visual search (see Introduction) it is unclear what kind of memory underlies search. In particular, the question whether it is memory for previously visited locations or memory for the identity of objects that guides search has recently been examined more closely. Beck, Peterson, and Vomela (2006) changed either the identity or the location of previously visited or new items during search to investigate whether these manipulations would distort search. Because changing the location rather than changing the identity of items disrupted the search process, they concluded that the memory representation that guides search is based more on location information. This result does not necessarily preclude the possibility that participants have the capacity to retain identity information during search, but simply do not use it. Together, these results suggest that participants have the capacity to store the identity of the distractors, but that, when the display changes for each search, this information is not encoded. This failure to encode identity may be the exception rather than the rule. For example, Zelinsky and Loschky (2005) had participants free-view nine-item scenes. Participants showed recognition memory above chance for objects which they had seen up to seven fixations before the recognition test. The percentage of correct object identifications in a test phase depended on the number of intervening fixations and resulted in a recency effect that was very similar to ours. A similar approach was used by Beck, Peterson, Boot, Vomela, and Kramer (2006) to study explicit object memory in search. On $33 \%$ of the trials, they ended a trial prematurely and asked participants to identify which of two items had been presented at a 
specific location. They found that participants performed above chance for about the last four fixated items and that performance decreased with item recency. Taken together, these results suggest that observers acquire identity memory for objects while inspecting or searching a display and that this memory can be deployed when probed explicitly and also to guide ongoing search.

The present results indicate that short-term memory is involved in facilitating subsequent search (see Gilchrist \& Harvey, 2000; McCarley et al., 2003, for similar arguments for a single search) and that the identity of distractors can be encoded. Like other short-term memory systems in the literature, this system appears to have limited capacity.

\section{AUTHOR NOTE}

Preparatory work was supported by grants from the European Community (HPMF-CT-2000-00986) to C.K. and I.D.G. and from the Wellcome Trust to I.D.G. Data collection was supported by Grant P17458-G04 of the Austrian Science Fund (FWF) to C.K. We are grateful to Margit Höfler and Barbara Tröbinger for collecting the data and to Jeremy Wolfe for helpful comments on an earlier version of this article. Correspondence regarding this article should be addressed to C. Körner, Institut für Psychologie, University of Graz, Universitätsplatz 2/III, A-8010 Graz, Austria (e-mail: christof.koerner@uni-graz.at).

\section{REFERENCES}

Atkinson, R. C., \& Shiffrin, R. M. (1968). Human memory: A proposed system and its control processes. In K. Spence \& J. Spence (Eds.), The psychology of learning and motivation (Vol. 2). New York: Academic Press.

Beck, M. R., Peterson, M. S., Boot, W. R., Vomela, M., \& Kramer, A. F. (2006). Explicit memory for rejected distractors during visual search. Visual Cognition, 14, 150-174.

Beck, M. R., Peterson, M. S., \& Vomela, M. (2006). Memory for where, but not what, is used during visual search. Journal of Experimental Psychology: Human Perception \& Performance, 32, 235-250.
Boot, W. R., McCarley, J. M., Kramer, A. F., \& Peterson, M. S. (2004). Automatic and intentional memory processes in visual search. Psychonomic Bulletin \& Review, 5, 854-861.

Bouma, H. (1970). Interaction effects in parafoveal letter recognition. Nature, 226, 177-178.

Cowan, N. (2001). The magical number 4 in short-term memory: A reconsideration of mental storage capacity. Behavioral \& Brain Sciences, 24, 87-114.

Gilchrist, I. D., \& HARVEY, M. (2000). Refixation frequency and memory mechanisms in visual search. Current Biology, 10, 1209-1212.

Horowitz, T. S., \& WolfE, J. M. (1998). Visual search has no memory. Nature, 394, 575-577.

KLEIN, R. M., \& MCINNES. W. J. (1999). Inhibition of return is a foraging facilitator in visual search. Psychological Science, 10, 346-352.

Körner, C., \& Gilchrist, I. D. (in press). Memory processes in multiple-target visual search. Psychological Research.

Kristjansson, A. (2000). In search of remembrance: Evidence for memory in visual search. Psychological Science, 11, 328-332.

McCarley, J. S., Wang, R. F., Kramer, A. F., Irwin, D. E., \& PeTERSON, M. S. (2003). How much memory does occulomotor search have? Psychological Science, 14, 422-426.

McCrary, J., \& Hunter, W. S. (1953). Serial position curves in verbal learning. Science, 117, 131-137.

Peterson, M. S., Kramer, A. F., Wang, R. F., Irwin, D. E., \& McCarLEY, J. S. (2001). Visual search has memory. Psychological Science, 12, 287-292.

Shore, D. I., \& KleIN, R. M. (2000). On the manifestations of memory in visual search. Spatial Vision, 14, 59-75.

Wolfe, J. M., Klempen, N., \& Dahlen, K. (2000). Postattentive vision. Journal of Experimental Psychology: Human Perception \& Performance, 26, 693-716.

Wolfe, J. M., Oliva, A., Butcher, S. J., \& Arsenio, H. C. (2002). An unbinding problem? The disintegration of visible, previously attended objects does not attract attention. Journal of Vision, 2, 256-271.

Zelinsky, G. J., \& LoschKy, L. C. (2005). Eye movements serialize memory for objects in scenes. Perception \& Psychophysics, 67, 676-690.

(Manuscript received June 2, 2006;

revision accepted for publication November 22, 2006.) 